\title{
Tests for Spatial Correlation of Dependent Variables in Spatial Dynamic Panel Data Models
}

\author{
Rong Sun* \\ Dept of Math and statistics, Chongqing Universty of Technol and Business, Chongqing, 400067, China \\ *Corresponding author
}

\begin{abstract}
This paper considers a spatial dynamic panel data regression model with fixed effects, spatial correlation of dependent variables and error serial among cross sectional units. When the number of individuals $n$, the number of time periods $T$ are large, and $T$ is asymptotically large relative to $n$, the paper derives various Lagrange multiplier tests and likelihood ratio test statistics for this panel data regression model including tests for spatial correlation of dependent variables in jointly, marginally or conditionally (one -dimensional and two-dimensional). Limiting null distributions of the tests are derived.
\end{abstract}

Keywords-dynamic spatial panel data; spatial correlation; lagrange multiplier tests; likelihood ratio tests

\section{INTRODUCTION}

Spatial panel models are becoming popular in econometric applications. To capture correlation among cross-sectional units, the spatial autoregressive (SAR) model by Cliff and Ord (1973) has received the most attention in economics. The texting for SAR models, Baltagi and Li (1995) addressed the problem of jointly testing for error serials correlation and individual effects for the panel data model with no spatial effects; Anselin (1988, 2001) and Anselin and Bera (1998), have studied the problem of testing for spatial dependence; Baltagi et al. (2003) considered the problem of jointly testing for random region effects in the panel as well as spatial correlation of error serials across these regions. however, the study did not consider the added problem of serial correlation in the remainder error term. Baltagi et al. (2007) consider the testing of spatial and serial dependence in an extended model, where serial correlation on each spatial unit over time, in addition to spatial dependence across spatial units are allowed in the disturbances; Myoung Jin Jang and Dong Wan Shin(2014) consider the texting for time effects and spatial effects by Lagrange multiplier and likelihood ratio texts about panel model with spatial autocorrelation and heterogeneity of error serials across time, they derived the limiting null distributions of test statistics jointly, marginally or conditionally. These panel models do not incorporate time lagged dependent variables as dynamic structures in the regression equation, this paper shall generalizes the previous studies by deriving joint, conditional and marginal tests of LM and LR, that consider the texting of the lag effects on dependent variables, spatial and serial dependence in the extended dynamic panel model with spatial correlation of dependent variables and error serial among cross sectional units, and studies their small sample properties using Monte Carlo experiments, our Monte Carlo evidence shows their good size properties and power properties except for a few cases where there is significant size distortion and the lower power.

The remainder of the paper is organized as follows. Section 2 presents a spatial dynamic panel data regression model with fixed effects and spatial correlation of dependent variables cross sectional units. Section 3 provides the LM and LR test statistics as well as their limiting null distributions.

\section{THE MODEL AND THE LIKELIHOOD FUNCTION}

\section{A. The Model}

The regression model Considered in this paper is

$$
\begin{gathered}
Y_{n t}=\lambda_{0} W_{n} Y_{n t}+\gamma_{0} Y_{n, t-1}+X_{n t} \beta_{0}+C_{n 0}+U_{n t} \\
U_{n t}=\rho_{0} M_{n} U_{n t}+V_{n t} \\
t=1,2, \ldots T
\end{gathered}
$$

where

$$
Y_{n t}=\left(y_{1 t}, y_{2 t}, \ldots y_{n t}\right)
$$

$V_{n t}=\left(v_{1 t}, v_{2 t}, \ldots v_{n t}\right)$ are $n \times 1$ column vector and $v_{i t}$ is i.i.d across $i$ and $d$ with zero mean and variance $\sigma_{0}^{2} W_{n}$ is an $n \times n$ nonstochastic spatial weights matrix that generates the spatial dependence on $y_{i t}$ between cross sectional units, $X_{i t}$ is the p-dimensional vector of covariates, and $C_{n 0}$ is an $n \times 1$ column vector of fixed effection. $M_{n}$ is an $n \times n$ nonstochastic spatial weights matrix for the disturbance.

Therefore, the parameters in this model is $\left(\lambda, \gamma, \beta^{\prime}, \rho, \sigma^{2}\right)$. if unnecessary, will make the inference complicated and even inefficient when $T$ or $N$ is fixed.

Define $S(\lambda)=I_{n}-\lambda W_{n}$ and $R(\rho)=I_{n}-\rho M_{n}$ for any $\lambda$ and $\rho$ at the true parameter Assuming the infinite sums are well-defined, by continuous substitution,

$$
Y_{n t}=\sum_{h=1}^{\infty}\left(S_{n}^{-1}\right)^{h} \gamma^{h-1}\left(C_{n 0}+X_{n, t-h} \beta_{0}+\left(R_{n}^{-1}\right)^{h-1} V_{n, t-h}\right)
$$




$$
=A_{n}+\mathrm{X}_{n t} \beta_{0}+E_{n}
$$

Where

$$
\begin{aligned}
& A_{n}=\sum_{h=1}^{\infty}\left(S_{n}^{-1}\right)^{h} \gamma^{h-1} C_{n 0} \quad, \quad X_{n t}=\sum_{h=1}^{\infty}\left(S_{n}^{-1}\right)^{h} \gamma^{h-1} X_{n, t-h} \\
& E_{n}=\sum_{h=1}^{\infty}\left(S_{n}^{-1}\right)^{h}\left(\gamma R_{n}^{-1}\right)^{h-1} V_{n, t-h}
\end{aligned}
$$

\section{B. The Concentrated Likelihood Function}

Denote $\theta=\left(\lambda, \gamma, \rho, \sigma^{2}\right), \theta_{0}$ indicates the true value of parameters. For notational purpose,we define $\tilde{Y}_{n t}=Y_{n t}-\frac{1}{T} \sum_{t=1}^{T} Y_{n t}, \bar{Y}_{n T,-1}=\frac{1}{T} \sum_{t=1}^{T} Y_{n, t-1} \quad S=S_{n}(\lambda)$, $\hat{S}=S_{n}(\hat{\lambda}) \quad, \quad \hat{S}=S_{n}(\hat{\lambda}) \quad . \quad \tilde{Y}=\left(\tilde{Y}_{n 1}^{\prime}, \ldots, \tilde{Y}_{n T}^{\prime}\right)^{\prime}$, $\tilde{Y}_{-1}=\left(\tilde{Y}_{n 0}^{\prime}, \ldots, \tilde{Y}_{n T-1}^{\prime}\right)^{\prime}$. Where $\hat{\lambda}_{\text {is unrestricted QMLES, }}$ $\lambda$ is restricted QMLES, Similarry, we define $\tilde{X}_{n t}, \tilde{V}_{n t}, R, \hat{R}, \tilde{R}, \tilde{X}, \tilde{X}_{-1}, \tilde{X}, \tilde{X}_{-1}$.

By the transformation approach we get the likelihood function is

$$
\begin{gathered}
L_{n T}(\theta)=-\frac{n(T-1)}{2} \ln 2 \pi-\frac{n(T-1)}{2} \ln \sigma^{2}+(T-1)(\ln |S|+\ln |R|)-\frac{1}{2 \sigma^{2}} \sum_{t=1}^{T} V_{n t}^{\tilde{1}} \tilde{V}_{n t} \\
\text { Where } \tilde{V}_{n t}=R\left[S \tilde{Y}_{n t}-\gamma \tilde{Y}_{n, t-1}-\tilde{X}_{n t} \beta\right]
\end{gathered}
$$

\section{TEST STATISTICS}

In this section, we derive $L M$ tests and $L R$ tests as well as their limiting null distributions. The LM statistic for testing a specific null hypothesis $H_{0}$ against $H_{1}$ is given by

$$
L M=\left(\left.\frac{\partial L_{n T}}{\partial \theta_{*}}\right|_{\theta_{*}=\tilde{\theta}_{*}}\right)^{\prime}\left[\left.E\left(\frac{\partial^{2} L_{n T}(\theta, \beta)}{\partial \theta_{*} \partial \theta_{*}^{\prime}}\right)\right|_{\theta_{*}=\tilde{\theta}_{*}}\right]^{-1}\left(\left.\frac{\partial L_{n T}}{\partial \theta_{*}}\right|_{\theta_{*}=\tilde{\theta}_{*}}\right)(5)
$$

Where $\theta_{*}=(\lambda, \gamma, \rho)^{\prime}, \quad \tilde{\theta}_{*}$ is the restricted QMLES under $\quad H_{0} \quad$,and $\left.\quad \frac{\partial L_{n T}(\theta, \beta)}{\partial \theta_{*}}\right|_{\theta_{*}=\tilde{\theta}} \quad$ and $\left.\frac{\partial^{2} L_{n T}(\theta, \beta)}{\partial \theta_{*} \partial \theta_{*}^{\prime}}\right|_{\theta_{*}=\tilde{\theta}}$ are the partial derivatives with respect to $\theta$, evaluated at $\tilde{\theta}$.The $L R$ statistic is given by $L R=L(\hat{\theta})-L(\tilde{\theta})$, where $\hat{\theta}$ is the unrestricted MLE. For simplicity of notation, let

$$
I(\theta)=E\left(\partial^{2} L_{n T}(\theta, \beta) / \partial \theta \partial \theta^{\prime}\right), \quad L_{U}=L(\hat{\theta}) \text { and }
$$

$$
L_{R}=L(\tilde{\theta})
$$

IV TESTS FOR SPATIAL CORRELATION ON $y_{i t}$ BETWEEN

$$
\text { CROSS SeCtional Units } H_{0}: \lambda=0
$$

We derive marginal and conditional tests for $s$ for spatial correlation on $y_{i t}$ between cross sectional units.

A. One-dimensional Conditional Tests for Spatial

Correlation $H_{0}: \lambda=0$ Assuming Admitting Non-zero $\gamma$ and $\rho$

Under $H_{0}$

$$
\begin{gathered}
\tilde{Y}_{n t}=\sum_{h=0}^{\infty} \gamma^{h}\left(\tilde{X}_{n, t-h} \beta_{0}+R_{n}^{-1} \tilde{V}_{n, t-h}\right) \\
=\tilde{X}_{n t} \beta+R_{n}^{-1} E_{n t}
\end{gathered}
$$

Where, $\tilde{X}_{n t}=\sum_{h=0}^{\infty} \gamma^{h} \tilde{X}_{n, t-h}, E_{n t}=\sum_{h=0}^{\infty} \gamma^{h} \tilde{V}_{n, t-h}$ Under $H_{0}$, we have

$$
\left.\frac{\partial L_{n T}}{\partial \theta_{*}}\right|_{\theta_{*}=\tilde{\theta}_{*}}=\left(\begin{array}{c}
D(\tilde{\lambda}) \\
0 \\
0
\end{array}\right)=\left(\begin{array}{c}
\frac{1}{\tilde{n}} \sum_{t=1}^{T}\left(\tilde{R} W \tilde{Y}_{n t}\right)^{\prime} \tilde{V}_{n t} \\
0 \\
0
\end{array}\right)
$$

the elements of the information matrix $\left.I\left(\hat{\theta}_{*}\right)\right|_{H_{0}} ^{c}=E\left(\left.\frac{\partial^{2} L_{n T}(\theta, \beta)}{\partial \theta_{*} \partial \theta_{*}^{\prime}}\right|_{\theta_{*}=\tilde{\theta}} ^{c}\right.$ are given by

$\left.I_{11}\right|_{H_{0}} ^{d}=(T-1) t r W^{2}+\hat{A_{t}} \operatorname{tr}\left(R^{-1} W^{\prime} \hat{R}^{\prime} \hat{R} W R^{-1}\right)+\frac{1}{\sigma^{2}} \hat{\beta}^{\prime} \sum_{t=1}^{T} \tilde{X}_{n t}^{\prime} W^{\prime} \hat{R}^{\prime} \hat{R} W \tilde{X_{n t}} \hat{\beta}$

$$
\left.I_{12}\right|_{H_{0}} ^{d}=\frac{1}{\sigma^{2}} \hat{\beta}^{\prime} \sum_{t=1}^{T} \tilde{X}_{n t}^{\prime} W^{\prime} \stackrel{\cap}{R}^{\prime} \stackrel{\cap}{R} W X_{n t-1}^{\sim} \hat{\beta}
$$

$$
\left.I_{13}\right|_{H_{0}} ^{d}=\hat{B}_{\lambda}\left[T-1-\frac{\hat{\left(\gamma-\hat{\gamma^{T}}\right)}}{(1-\hat{\gamma})\left(1-\hat{\gamma^{2}}\right)}+\frac{\sum_{i=1}^{T-1} i \hat{\gamma}^{i}}{\left(1-\gamma^{2}\right) T}\right]
$$




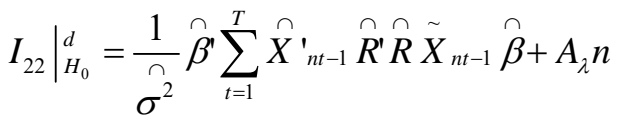

$$
\begin{aligned}
& \left.I_{23}\right|_{H_{0}} ^{d}=-2\left[\frac{(T-1)-(T-1)\left(\hat{\gamma}-\hat{\gamma}^{T-1}\right)}{T(1-\hat{\gamma})}-\frac{\sum_{i=1}^{T-2} i \hat{\gamma}^{i}}{T}\right] \operatorname{tr}\left(M \stackrel{\cap^{-1}}{R}\right) \\
& \left.I_{33}\right|_{H_{0}} ^{c}=(T-1) \operatorname{tr}\left[\hat{H}_{n}^{\prime} \hat{H}_{n}+\hat{H}_{n}^{2}\right]
\end{aligned}
$$$$
\text { Where } \quad \hat{A_{\lambda}}=\left[\frac{T-1}{\left(1-\gamma^{2}\right)}-\frac{2\left(1-\hat{\gamma}^{T}\right)}{\hat{\imath}(1-\hat{\gamma})\left(1-\gamma^{2}\right)}+\frac{2 \sum_{i=1}^{T-1} i \hat{\gamma}^{i}}{T\left(1-\gamma^{2}\right)}\right]
$$$$
{\stackrel{\cap}{B_{\lambda}}}=\operatorname{tr}\left[\stackrel{\cap}{R}^{-1} W M+\left(\stackrel{\cap}{R}^{-1}\right)^{2} W \stackrel{\cap}{R} M\right]
$$

$$
\begin{aligned}
& \hat{\beta}=\left(\tilde{X}_{n}^{\prime} \stackrel{\hat{R}}{R^{\prime}} \hat{R} \tilde{X}_{n}\right)^{-1} \tilde{X}_{n}^{\prime} \stackrel{\imath}{R^{\prime}} \stackrel{\cap}{R}\left(\tilde{Y}_{n t}-\tilde{\gamma} \tilde{Y}_{n t-1}\right), \\
& \tilde{\tilde{V}}_{n t}=\hat{R}\left(\tilde{Y}_{n t}-\hat{\gamma} \tilde{Y}_{n, t-1}-\tilde{X}_{n t} \hat{\beta}\right), \hat{\sigma}^{2}=\sum_{t=1}^{T} \tilde{V}_{m}^{\prime} \tilde{V}_{n t} / n(T-1)
\end{aligned}
$$

So, by equation (5) the resulting conditional LM statistic is given

$$
L M_{i}=D(\hat{\lambda})^{2} /\left\{(T-1) r W^{2}+A_{t} t r\left(R^{-1} W^{\prime} \hat{R}^{\prime} \hat{R} W^{-1} R^{-1}\right)+\frac{1}{\sigma^{2}} \hat{\beta} \sum_{i=1}^{T} \hat{X}_{W=1}^{\prime} W^{\prime} \hat{R} \hat{R} W \hat{X}_{n-1} \hat{\beta}\right\}
$$

The derivation of this $L M$ test statistic is given in Appendix A.3. This $L M$ statistic should be asymptotically distributed as $\chi_{1}^{2}$ under $H_{0}$ as $T, N \rightarrow \infty$ which will be formally proved in Theorem 3 in Section 3.6.

The conditional $L R$ test $\mathrm{t}$ for $H_{0}: \lambda=0$ vs $H_{1}: \lambda \neq 0$ admitting non-zero $\lambda$ and $\rho$ is given by $L R_{\lambda}=2\left(L_{U}-L_{R}\right)$

Where

$$
\begin{gathered}
L_{U}=-\frac{n(T-1)}{2}\left(\ln 2 \pi \hat{\sigma}^{2}+1\right)+(T-1)(\ln |\hat{S}|+\ln |\hat{R}|), \\
L_{R}=-\frac{n(T-1)}{2}\left(\ln 2 \pi \hat{\sigma}^{2}+1\right)+(T-1) \ln |\hat{R}|
\end{gathered}
$$

Where the unrestricted MLEs are same as equation (9).

$$
\begin{gathered}
\text { Under } H_{0} \text { the } L R_{\lambda} \text { statistic is asymptotically } \\
\text { distributed as } \chi_{1}^{2}
\end{gathered}
$$

\section{B. One -dimensional Marginal Tests for Spatial Error}

Correlation $H_{0}: \lambda=0$ Assuming $\gamma=\rho=0$

This is a one-dimensional marginal test for spatial dependence on $y_{i t}$ no ignoring the presence of lag effect and spatial error correlation cross sectional units. when $\gamma=\rho=0$, $R$ reduce to unit matrix , under the null hypothesis,

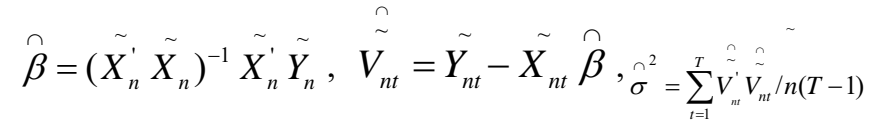
$D(\hat{\lambda})=\frac{1}{\hat{\sigma}^{2}} \sum_{t=1}^{T}\left(W \tilde{Y}_{n t}\right)^{\prime} \tilde{V}_{n t}^{\prime},\left.\quad I_{11}\right|_{H_{0}} ^{c} 2(T-1) t r W^{2}+\frac{1}{\sigma^{2}} \hat{\beta}^{\prime} \sum_{t=1}^{T} \tilde{X}_{n t}^{\prime} W^{\prime} W \tilde{X}_{n t} \hat{\beta}$ the LM test statistic for testing $H_{0}$, call it $L M_{\lambda / \gamma \rho}$

$$
L M_{\lambda / \not p}=D(\hat{\gamma})^{2} /\left(2(T-1) t r W^{2}+\frac{1}{\sigma^{2}} \beta \sum_{t=1}^{T} X_{m}^{\prime} W^{\prime} W \tilde{X}_{n t} \hat{\beta}\right) \hat{W}_{n t} / n(T-1)
$$

This LM statistic should be asymptotically distributed as $\chi_{1}^{2}$ under $H_{0}$ as as $T, n \rightarrow \infty$ which will be formally proved in Theorem 2 in Section 3.5.

The Marginal $L R$ test for $H_{0}: \gamma=0$ vs $H_{1}: \gamma \neq 0$ assuming $\lambda=\rho=0$ is given by $L R_{\lambda / \gamma \rho}=2\left(L_{U}-L_{R}\right)$

$$
L_{U}=-\frac{n(T-1)}{2}\left(\ln 2 \pi \hat{\sigma}^{2}+1\right) \quad L_{R}=-\frac{n(T-1)}{2}\left(\ln 2 \pi \dot{\sigma}^{2}+1\right)
$$

Where

$$
\hat{\beta}=\left(\tilde{X_{n}^{\prime}} \tilde{X}_{n}\right)^{-1} \tilde{X}_{n}^{\prime}\left(\tilde{Y}_{n t}-\hat{\gamma} \tilde{Y}_{n t-1}^{\sim}\right)
$$

$\hat{\tilde{V}}_{n t}=\tilde{Y}_{n t}-\hat{\gamma} \tilde{Y}_{n t-1}-\tilde{X}_{n t-1} \hat{\beta}, \hat{\sigma}^{2}=\sum_{t=1}^{T} \hat{V}_{n t}^{\prime} \hat{\tilde{V}}_{n t} / n(T-1)$,

Under $H_{0}$ the $L R_{\lambda / \gamma \rho}$ statistic is asymptotically
distributed as $\chi_{1}^{2}$

\section{SUMMARY}

This paper generalized the model in Baltagi and Li (1995) paper, Baltagi et al. (2003) paper and Baltagi et al. (2007) paper, and considers a spatial dynamic panel data regression model with fixed effects, spatial correlation of dependent variables and error serial among cross sectional units. When the number of individuals $\mathrm{n}$, the number of time periods $\mathrm{T}$ are large, and $\mathrm{T}$ is asymptotically large relative to $\mathrm{n}$, the paper derives various Lagrange multiplier tests and likelihood ratio test statistics for this panel data regression model including tests for spatial correlation of dependent variables. Limiting null distributions of the tests are derived. 


\section{ACKNOWLEDGEMENT}

This research was financially supported by by Statistical science research project of China National Bureau of Statistics (2016LY28) and Social science planning project of Chongqing Social Science Association (2016YBJJ022).We would like thank anonymous referees for their helpful comments and suggestions.

\section{REFERENCES}

[1] Cliff, A.D., Ord, J.K., 1973. Spatial Autocorrelation. Pion Ltd., London.

[2] Baltagi, B.H., Li, Q., 1995. Testing AR (1) against MA (1) disturbances in an error component model. Journal of Econometrics 68, 133-151.

[3] Anselin, L., Bera, A.K., 1998. Spatial dependence in linear regression models with an introduction to spatial econometrics. In: Ullah, A., Giles, D.E.A. (Eds.), Handbook of Applied Economic Statistics. Marcel Dekker, New York.

[4] Anselin, L., 2001. Rao's score tests in spatial econometrics. Journal of Statistical Planning and Inference 97, 113-139.

[5] B.H. Ba ltagi, S.H. Song, and W. Koh, Testing panel data regression models with spatial error correlation, J. Econometrics 117 (2003), 123-150.

[6] Baltagi, B., Song, S.H., Jung, B.C., Kon, W., 2007. Testing for serial correlation, spatial autocorrelation and random effects using panel data. Journal of Econometrics 140, 5-51.

[7] Myoung Jin Jang and DongWan Shin, Tests for random time effects and spatial error correlation in panel regression models. Statistics, 48(2014), No. $1,101-120$.

[8] Jihai Yua, Robert de Jong, Lung-fei Lee, Quasi-maximum likelihood estimators for spatial dynamic panel data with fixed effects when both $\mathrm{n}$ and $\mathrm{T}$ are largeI, Journal of Econometrics 146 (2008), 118-134.

[9] Lee, L.F., and J. Yu, Estimation of Spatial Autoregressive Panel Data Models with Fixed Effects. Journal of Econometrics. Journal of Econometrics 154(2010), 165-185.

[10] Su L, Yang Z, Su L, et al. QML estimation of dynamic panel data models with spatial errors. Working Paper [J]. Journal of Econometrics, 2007, 185(1):230-258 